\title{
On the use of time-dependent filters for large-eddy simulation
}

\author{
Pierre Sagaut \\ ONERA/DSNA, 29 av. Division Leclerc, \\ 92 Châtillon, France \\ sagaut@onera.fr
}

\section{Introduction}

Large-Eddy Simulation (LES) [5] is a technique for the numerical simulation of three-dimensional unsteady turbulent flows. Direct numerical simulation of all the time-space scales present in the exact solution of the Navier-Stokes equations being too expensive, a scale selection is performed, the small scales (i.e. the high frequencies) being filtered out of the computed solution. ¿From a mathematical point of view, the filtering is represented through the application of a convolution filter to the exact solution $u(x, t)$ :

$$
\bar{u}(x, t)=G \star u=\int_{\Omega_{f}} G(x-y, \Delta) u(y, t) d y
$$

where $\Omega_{f}$ and $\Delta$ are the domain occupied by the fluid and the characteristic lengthscale of the filter, respectively. Basic equations for LES are obtained by applying the same filter to the Navier-Stokes equations. Some mathematical requirements can be defined on the filter in order to ensure that it commutes with the time- and space-derivatives, leading to tractable forms of the filtered Navier-Stokes equations. Ghosal and Moin [4], van der Ven [6] and more recently Vasyliev et al. [7] worked on that subject. The latter authors have defined some stationary filters which commute up to an arbitrary order with space derivatives in the case of non-homogeneous filters (i.e. $\Delta=\Delta(x)$ ) applied on bounded domains. Time-dependent filter have not been considered up to now.

The scope of the present work is to derive the basic equations for LES for fluid-structure problems, using the Arbitrary Lagrangin-Eulerian (ALE) technique for the fluid part of the algorithm. Use of ALE yields time-dependent computational grids. The filter length $\Delta$ being associated to the local mesh size, it can no longer be considered as a time-independent variable (i.e. $\Delta=\Delta(x, t)$ ), and its time-variations must be explicitely taken into account.

The paper is organized as follows : section 2 presents the problem for time-dependent filters. Basic equations for the ALE/LES problem with typ-

Article published by EDP Sciences and available at http://www.edpsciences.org/proc or http://dx.doi.org/10.1051/proc:2001015 
ical boundary conditions for the fluid-structure interaction problem are derived in section 3. Conclusions and perspectives are given in section 4.

\section{Basic equations for time-dependent filter}

We consider the Navier-Stokes equations for an incompressible fluid on a static grid :

$$
\begin{gathered}
\frac{\partial u}{\partial t}+C(u, u)+\nabla p-B(u)=0 \\
\nabla \cdot u=0
\end{gathered}
$$

where $C(u, u)$ is the bilinear form associated to the convection term, and $B(u)$ the linear form associated to the diffusion term :

$$
C(u, v)=u \nabla v, \quad B(u)=\nu \nabla^{2} u
$$

with $p$ and $\nu$ the static pressure and the kinematic viscosity, respectively. Introducing the commutation error operator [.,.] :

$$
[f, g](u)=f \circ g(u)-g \circ f(u)
$$

the filtered Navier-Stokes equations can be written as :

$$
\begin{array}{r}
\frac{\partial \bar{u}}{\partial t}+C(\bar{u}, \bar{u})+\nabla \bar{p}-B(\bar{u})= \\
\left.\left[\frac{\partial}{\partial t}, G \star\right](u)+[C(., .), G \star](u, u)+[\nabla, G \star](p)-[B(.), G \star)\right](u) \\
\nabla \cdot \bar{u}=[\nabla \cdot, G \star](u)
\end{array}
$$

These two equations make it possible to do several remarks :

1. All the commutation error terms are reported in the right hand side of the equations. The first term in the rhs of Eq.(4) comes from the timedependency of the filter. The second term corresponds to a generalized form of the usual subgrid force, because it includes commutation error with the gradient operator:

$$
\begin{aligned}
{[C(., .), G \star](u, u) } & =\bar{u} \nabla \bar{u}-\overline{u \nabla u} \\
& =\bar{u} \overline{\nabla u}-\overline{u \nabla u}+\bar{u}[\nabla, G \star](u)
\end{aligned}
$$

2. The divergence of the filtered velocity field is not a priori equal to zero 
3. The viscosity is constant and uniform, then

$$
[B(.), G \star](u)=\nu\left[\nabla^{2}, G \star\right](u)
$$

The commutation error between the filter and spatial derivatives can be expressed for a dummy variable $\phi$ as [3, 2] :

$$
\left[\frac{\partial}{\partial x}, G \star\right](\phi)=\left(\frac{\partial G}{\partial \Delta} \star \phi\right) \frac{\partial \Delta}{\partial x}+\int_{\partial \Omega_{f}} G(x-y, \Delta(x, t)) \phi(y, t) n(y) d s
$$

where $n$ is the outward unit normal vector to the boundary of $\Omega_{f}, \partial \Omega_{f}$. Filter kernels $G$ such that this commutation error is $O\left(\Delta^{l}\right)$, with arbitrary $l$, are given in Ref.[7].

We now consider the commutation error with the time derivative. A general form of this error is :

$$
\left[\frac{\partial}{\partial t}, G \star\right](\phi)=\frac{\partial \Delta}{\partial t}\left(\frac{\partial G}{\partial \Delta} \star \phi\right)+\frac{\partial G}{\partial t} \star \phi
$$

Two contributions appear : the first one is associated to the time-variation of the filtering length (mesh size), and the second one accounts for a possible explicit dependency of the filter kernel with respect to time. The form of the filter being generally linked to the numerical scheme, the latter contribution would correspond to a change in the numerical method during the computation. This case will not be considered in the following, and that part of the commutation error will be assumed to be zero.

Acceptable filters will be such that the commutation error with time derivative is at least of the same order as the commutation error with space derivatives, i.e.

$$
\left\|\left[\frac{\partial}{\partial x}, G \star\right](u)\right\|=O\left(\Delta^{l}\right),\left\|\left[\frac{\partial}{\partial t}, G \star\right](u)\right\|=O\left(\Delta^{m}\right), m \geq l
$$

\section{Towards an ALE/LES problem}

\subsection{Filtered ALE equations}

We now extend the analysis to the case of the ALE equations with boundary conditions associated to the fluid-structure interaction problem. The basic set of unfiltered equations reads

$$
\begin{gathered}
\frac{\partial u}{\partial t}+C(u-w, u)+\nabla p-B(u)=0 \\
\nabla \cdot u=0
\end{gathered}
$$

where $w$ is the velocity associated to the mesh, i.e. the grid nodes move at the $w$ velocity. 
The filtered ALE equations are obtained by applying a convolution filter, exactly in the same way as for the usual Navier-Stokes equations, yielding

$$
\begin{array}{r}
\frac{\partial \bar{u}}{\partial t}+C(\bar{u}-\bar{w}, \bar{u})+\nabla \bar{p}-B(\bar{u})= \\
\left.\left[\frac{\partial}{\partial t}, G \star\right](u)+[C(., .), G \star](u-w, u)+[\nabla, G \star](p)-[B(.), G \star)\right](u)
\end{array}
$$

The only difference with the usual filtered momentum equation (4) appears in the convection term and the associated commutation error term in the rhs of Eq.(10). The filtered continuity equation is exactly the same as in the NavierStokes case.

Using the linearity property, the new convective commutation error term can be written as

$$
[C(., .), G \star](u-w, u)=[C(., .), G \star](u, u)-[C(., .), G \star](w, u)
$$

This splitted formulation show clearly that, in the particular case of a pure Lagrangian numerical scheme $(w \equiv u)$ the subgrid terms are only generated by the commutation error between the derivatives and the filter. In the case of a perfectly commuting filter, no subgrid terms appear at all.

\subsection{Control of the commutation error with time-derivative}

The control of the commutation error with the time-derivative operator makes it possible to define some constraint on the mesh velocity $w$, since the filter length $\Delta$ can be linked to the mesh size. The error term appears as the product of two terms. The first one is the variation rate of the filter length, $\partial \Delta / \partial t$. The second one is the convolution with the gradient of the initial kernel, $\partial G / \partial \Delta$. This last term has already been studied by previous authors, because it also appears in the commutation error with space-derivatives. General kernels which permit an explicit control of this term have been proposed (e .g. van der Ven's filters [6]), and we will address here the control of the first term.

In the case of weakly anisotropic grids, Deardorff [1] proposed the following evaluation :

$$
\Delta=V^{1 / 3}
$$

where $V$ is the volume of the computational cell for finite volume/finite element methods, and the Jacobian for finite difference technique. For strongly anisotropic meshes, the following estimation holds :

$$
\Delta=h
$$

where $h$ is the size of the longest edge of the smallest parallelepipedic volume containing the computational cell. It is worth noting that, for weakly anisotropic meshes, we have $V \simeq h^{3}$. 
For weakly anisotropic meshes, we get :

$$
\frac{\partial \Delta}{\partial t}=\frac{1}{3} V^{-2 / 3} \frac{\partial V}{\partial t}
$$

Using the classical result

$$
\frac{\partial V}{\partial t}=V \nabla \cdot w
$$

we obtain

$$
\frac{\partial \Delta}{\partial t}=\frac{1}{3} V^{1 / 3} \nabla \cdot w=O(h) \nabla \cdot w
$$

Then, is is seen that the commutation error with time derivative will be identically zero if $w$ is solenoidal for weakly anisotropic grids. Weaker control is obtained by enforcing $\nabla \cdot w=O\left(h^{n}\right), n \geq 0$.

For strongly anisotropic grids, assuming that $h$ is the size of the edge aligned with the $i$ th axis of the cartesian reference frame, we have :

$$
\frac{\partial \Delta}{\partial t}=\frac{\partial h}{\partial t}=h \frac{\partial w_{i}}{\partial x_{i}}
$$

The commutation error can be driven to zero by chosing a velocity $w$ such that $\partial w_{i} / \partial x_{i}=0$.

\subsection{Boundary conditions for fluid-structure interaction}

We now address the problem of the boundary conditions associated to a fluid/structure interface $\Gamma \subset \partial \Omega f$. Let $d$ be the displacement of the structure, $n$ the inward normal vector on $\Gamma$ and $F_{\Gamma}$ the force applied by the fluid on the structure.

For the unfiltered problem, we have :

$$
\begin{aligned}
u & =\frac{\partial d}{\partial t} \\
F_{\Gamma} & =-p n+\nu\left(\nabla u+\nabla^{t} u\right) n
\end{aligned}
$$

The corresponding boundary conditions for the LES problem are obtained by applying a convolution filter, leading to :

$$
\begin{aligned}
\bar{u} & =\frac{\partial \bar{d}}{\partial t}+\left[G \star, \frac{\partial}{\partial t}\right](d) \\
\bar{F}_{\Gamma} & =-\left(\bar{p} \bar{n}+\tau_{p}\right)+\nu\left(\nabla \bar{u}+\nabla^{t} \bar{u}\right) \bar{n}+\tau_{u}
\end{aligned}
$$

where the subgrid terms $\tau_{p}$ and $\tau_{u}$ are expressed as (it is recalled that the subgrid variation $\phi^{\prime}$ of a dummy variable $\phi$ is defined as $\phi^{\prime}=\phi-\bar{\phi}$ ): 


$$
\begin{aligned}
\tau_{p} & =\overline{p n}-\bar{p} \bar{n} \\
& =\overline{\bar{p} n^{\prime}}+\overline{p^{\prime} \bar{n}}+\overline{p^{\prime} n^{\prime}}+(\overline{\bar{p}} \bar{n}-\bar{p} \bar{n}) \\
\tau_{u} & =\nu \overline{\left(\nabla u+\nabla^{t} u\right) n}-\nu\left(\nabla \bar{u}+\nabla^{t} \bar{u}\right) \bar{n} \\
& =\nu\left(\tau_{\nabla}(\nabla, u, n)+\tau_{\nabla}\left(\nabla^{t}, u, n\right)\right)
\end{aligned}
$$

where

$$
\begin{aligned}
\tau_{\nabla}(\nabla, u, n) & =\overline{\nabla u n}-\nabla \bar{u} \bar{n} \\
& =(\overline{\overline{\nabla u} \bar{n}}-\nabla \bar{u} \bar{n})+\overline{\overline{\nabla u} n^{\prime}}+\overline{(\nabla u)^{\prime} \bar{n}}+\overline{(\nabla u)^{\prime} n^{\prime}}
\end{aligned}
$$

It is worth remembering that only filtered variables (with an overline) are known during the LES computations, and that all the terms involving the total field or the subgrid part (with a prime) must be modeled. The preceding filtered boundary conditions make two sources of subgrid fluctuations arising : the first one is the fluctuations of both velocity and pressure fields at scales smaller that the filter lengthscale $\Delta$, denoted $u^{\prime}$ and $p^{\prime}$ respectively, the second one is the fluctuation of the interface at high wavenumber (term including $n^{\prime}$ ). No subgrid models have been proposed at present time for these subgrid terms.

But it can be emphasized that for problems dealing with classical aerospace engineering devices, the typical length of the deformation of the structure is much larger than those associated to the turbulence, yielding $n^{\prime} \approx 0$.

But for new technological systems such as MEMS, whose characteristic size is of the same order as those of turbulence, the problem remains fully open.

\section{Conclusions and perspectives}

Problems associated with the definition of time-dependent filters have been devised for the first time to the knowledge of the author. The commutation error with the time-derivative is expressed, and the problem of its control is addressed.

The case of an ALE/LES algorithm for future application to fluid-structure application is then considered. The corresponding basic equations and boundary conditions are derived. Some sufficient conditions on the mesh velocity are given to obtain an explicit control of the commutation error with the time-derivative.

Future works will deal with the modelling of the new subgrid terms arising in the ALE/LES system. Possible ways for that purpose are the homogenization technique and the deconvolution approach.

\section{Acknowledgements}

The author thanks Dr. F. Coquel for giving him the opportunity to attend CEMRACS 99 and to start working on the ALE/LES problem. 


\section{References}

[1] Deardorff, J.W. (1970): A numerical study of three-dimensional turbulent channel flow at large Reynolds numbers. J. Fluid Mech. 41, 453-465

[2] Fureby, C., Tabor, G. (1997): Mathematical and physical constraints on large-eddy simulations. Theoret. Comput. Fluid Dynamics 9, 85-102

[3] Ghosal, S. (1999): Mathematical and physical constraints on large-eddy simulation of turbulence. AIAA J. 37 (4), 425-433

[4] Ghosal, S., Moin, P. (1995): The basic equations for the large-eddy simulation of turbulent flows in complex geometry. J. Comput. Phys. 118, $24-37$

[5] Sagaut, P. (1998): Introduction à la simulation des grandes échelles pour les écoulements de fluide incompressible (in french). Springer-Verlag, Berlin.

[6] van der Ven, H. (1995): A family of large eddy simulation (LES) filters with nonuniform filter widths. Phys. Fluids 7(5), 1171-1172

[7] Vasilyev, O., Lund, T.S., Moin, P. (1998): A general class of commutative filters for LES in complex geometries. J. Comput. Phys. 146, 82-104 